\title{
Association of early sporulation genes with suggested developmental decision points in Streptomyces coelicolor A3(2)
}

\author{
Klas Flärdh, $†$ Kim C. Findlay and Keith F. Chater \\ Author for correspondence: Keith F. Chater. Tel: +44 1603 452571. Fax: +44 1603456844. \\ e-mail: chater@bbsrc.ac.uk
}

John Innes Centre, Norwich Research Park, Colney, Norwich NR4 7UH, UK
Cytological analysis of a series of Streptomyces coelicolor A3(2) mutants with disruptions of early sporulation (whi, for white aerial mycelium) genes in an isogenic background has provided new information about the role of whiH, and confirmed and extended previous knowledge about whiG, whiA and whiB. The characteristic straight aerial hyphae of whiG mutants contained normally spaced vegetative-like septa, while mutants in whiA or whiB had abnormally long and coiled aerial hyphae almost devoid of septation. whiG, whiA and whiB were all absolutely required for sporulation septation, and for all visible signs of nucleoid condensation and partitioning and other changes associated with later stages of sporulation. On the other hand, whiH appeared to enhance low basal levels of these processes. Thus, whiH mutant aerial hyphae were divided into loosely coiled fragments of variable sizes by what appeared to be a few sporulation septa. These fragments showed some spore-like characteristics and contained condensed and aberrantly partitioned nucleoids. whiG, whiA and whiB were epistatic to whiH on the criterion that they prevented such fragments from forming in double mutants. These spore-like features and the synthesis of clearly detectable levels of the whiE-directed grey spore pigment were not due to any residual activity of previously studied whiH alleles since they were retained by a constructed whiH null mutant. A model is presented that explains the mutant phenotypes by proposing two early developmental decision points involved in commitment to sporulation septation, one requiring whiG and the other requiring whiA and whiB.

Keywords: cell division, whi genes, aerial mycelium, checkpoints

\section{INTRODUCTION}

During growth of rod-shaped unicellular bacteria such as Escherichia coli and Bacillus subtilis, cell wall extension takes place on lateral walls, and every DNA replication cycle is followed by partitioning of the sibling chromosomes, coordinated with the ingrowth of a centrally located septum at the site of incipient cell separation (Donachie, 1993; Park, 1996). None of these observations holds for the vegetative growth of streptomycetes to generate a substrate mycelium : most cell wall growth is at the hyphal tips; sibling DNA molecules do not show clearcut partitioning; septation occurs far

†Present address: Dept of Cell and Molecular Biology, Uppsala University, Biomedical Center, Box 596, S-75124 Uppsala, Sweden.

Abbreviations: 7-AAD, 7-aminoactinomycin D; DAPI, 4',6-diamidino-2phenylindole; Fluo-WGA, fluorescein-conjugated wheat germ agglutinin. (typically about $20 \mu \mathrm{m}$ ) from growing tips to give rise to adjacent multigenomic compartments; and cell separation does not take place. Quasi-exponential growth is possible only because of the emergence of branches (Prosser \& Tough, 1991).

The situation for streptomycetes is further complicated by their reproductive strategy, with which this paper is concerned. From the older parts of the substrate mycelium, new branches grow into the air to form an aerial mycelium, which eventually generates long chains of unigenomic spores. In the extensively studied organism Streptomyces coelicolor A3(2), some aspects of aerial hyphal wall structure change after an initial growth period, because a typical aerial hypha has a straight stalk surmounted by a somewhat coiled apical region (Wildermuth, 1970; Wildermuth \& Hopwood, 1970). The onset of sporulation in such aerial hyphae 
requires a switch to a different mode of septation and chromosome partitioning from that in vegetative hyphae. The key cell division protein FtsZ assembles into a large number of rings (sometimes as many as 50), forming a regular ladder (Schwedock et al., 1997). The rings are the precursors of sporulation septa, which delimit prespore compartments into which single nucleoids are partitioned. Sporulation septa differ morphologically from vegetative septa, having a structure that leads to full separation of adjacent cells and not simply to hyphal crosswalls (Wildermuth \& Hopwood, 1970). Maturation of the spores involves induction of late sporulation genes, rounding up and thickening of the cell wall and synthesis of a grey polyketide spore pigment (for reviews, see Chater, 1989, 1993, 1998; Chater \& Losick, 1997).

Several so-called whi (white) mutants have been isolated which form sporulation-defective aerial hyphae lacking the dark grey spore pigment (Hopwood et al., 1970; Chater, 1972). Previous work indicated that mutations in whiG, whiA, whiB or whiH block development at early stages and prevent sporulation septation (Chater, 1972; Hopwood et al., 1970; McVittie, 1974), and more recently Schwedock et al. (1997) used immunofluorescence microscopy to show that whiG, whiB and whiH mutants fail to assemble ladders of FtsZ rings in aerial hyphae. Of these 'early' whi genes, whiG encodes an alternative sigma factor (Chater et al., 1989); whiA encodes a protein of unknown function with orthologues in several other Gram-positive bacteria (N. J. Ryding, J. A. Aínsa, N. Hartley, C. J. Bruton \& K. F. Chater, unpublished); whiB belongs to a group of genes unique to actinomycetes, which code for small highly charged and cysteine-rich proteins of unknown function (Davis \& Chater, 1992; J. Soliveri, J. Gomez, W. R. Bishai \& K. F. Chater, unpublished); and whiH encodes a member of the GntR family of transcription factors (Ryding et al., 1998). It would be of considerable interest to understand how these genes, directly or indirectly, interact with the cell division machinery to control sporulation septation.

Despite an early suggestion to the contrary (Chater, 1972), it has become increasingly clear that the phenotypes of early whi mutants do not directly correspond to intermediate stages of normal development of wild-type spore chains. Rather, it seems that certain growth and/or morphological processes continue in the mutants after the point at which normal development is blocked, leading to mutation-specific terminal phenotypes. With the exception of the work by Schwedock et al. (1997), previous descriptions of these phenotypes have utilized only phase-contrast microscopy or transmission electron microscopy and have mostly dealt with uncharacterized presumptive point mutations which may retain residual activity to various degrees (Chater, 1972, 1975; Hopwood et al., 1970; McVittie, 1974; Plaskitt \& Chater, 1995; Ryding et al., 1998). The availability of defined gene disruptions and the recent development of a technique for transformation with denatured chromosomal DNA (Oh \& Chater, 1997) have made it straightforward to construct a series of defined null whiG, whiA, whiB and whiH mutants in an isogenic background. We have analysed hyphal morphology, septation patterns and distribution of nuclear material in fully developed aerial mycelium of these mutants using scanning electron and fluorescence microscopy. Based on these analyses, the whiG, whiA and whiB genes can be associated with blocks at specific early stages of morphological development, which may represent developmental decision points in the sporulation process. Furthermore, it is shown that whiH affects a different stage of sporulation than previously thought, and is not absolutely required for some of the later sporulation processes.

\section{METHODS}

Bacterial strains and growth conditions. The strains of Streptomyces coelicolor A3(2) used in the main part of this study are listed in Table 1. The following white, nonsporulating derivatives of the wild-type were also used: C47, C63, C66, C78, C90, C96, C109, C119, C219, C220, C240, C250 and C251 (Chater, 1972; Hopwood et al., 1970). The mutations in C109, C119, C219 and C250 were genetically mapped to the whiH locus (Chater, 1972), and the mutations in these and the remaining strains were all complemented by cloned whiH DNA on the low-copy-number pIJ698 (Ryding et al., 1998; N. J. Ryding, personal communication). The whiH63, whiH109, whiH119 and whiH209 mutant alleles have been sequenced (Ryding et al., 1998). E. coli strain DH5 $\alpha$ (Hanahan, 1983) was used for plasmid construction, and ET12567 (MacNeil et al., 1992) was used for obtaining nonmethylated plasmid DNA (S. coelicolor cannot be transformed by methylated DNA; Kieser \& Hopwood, 1991) for transformation of Streptomyces. Media and general techniques for growth and transformation of bacterial strains and manipulation of DNA were as described for Streptomyces by Hopwood et al. (1985) and for E. coli by Sambrook et al. (1989). Surface cultures for studies of sporulation-related phenotypes were grown on minimal medium (MM; Hopwood et al., 1985) agar with $0.7 \%$ mannitol as carbon source.

Construction of $\Delta$ whiH::ermE mutation. Plasmid pIJ599 carries a SacI fragment containing whiH and surrounding sequences in a derivative of the $t s r$ (thiostrepton resistance)containing pDH5 vector (Hilleman et al., 1991; Ryding et al., 1998). The whiH promoter and the first half of the whiH coding region were deleted from this plasmid by digesting with MunI and BamHI and replacing the $497 \mathrm{bp}$ whiH fragment with an EcoRI-BglII fragment containing the ermE gene from plasmid pIJ4026 (Bibb et al., 1985), which confers resistance to erythromycin and lincomycin (Fig. 1). The resulting plasmid, pIJ6304, was passed through E. coli strain ET12567, denatured in alkali (Oh \& Chater, 1997) and used to transform $S$. coelicolor strain J1501 to lincomycin resistance $\left(200 \mu \mathrm{g} \mathrm{ml}^{-1}\right)$. Transformants were screened for thiostrepton sensitivity to indicate loss of the vector replicon, and the disruption of the whiH locus in those strains was confirmed by Southern blotting. All strains in which whiH had been replaced with $\Delta$ whiH::ermE showed a characteristic whiH phenotype (see this paper). The $\Delta w h i H:$ :ermE mutation from one such strain, J2409, was transferred to strain M145 by chromosomal DNA transformation to create strain J2408, as described below.

Transfer of gene disruption alleles. Total DNA was prepared from previously constructed whi disruption mutants (Table 1) 
Table 1. Streptomyces coelicolor $A 3(2)$ strains and plasmids used in this study

\begin{tabular}{|c|c|c|}
\hline $\begin{array}{l}\text { Strain or } \\
\text { plasmid }\end{array}$ & Genotype & Reference \\
\hline \multicolumn{3}{|l|}{ Strains } \\
\hline C119 & whiH119 SCP1 ${ }^{+} \mathrm{SCP}^{+} \mathrm{Pgl}^{+}$ & Chater (1972) \\
\hline J1501 & bisA1 uraA1 strA1 $\mathrm{SCP}^{-} \mathrm{SCP}^{-} \mathrm{Pgl}^{-}$ & Chater et al. (1982) \\
\hline J1862 & J1501 whiG: :byg & C. Whatling \\
\hline $\mathrm{J} 2221$ & J1501 whiB:: hyg & Oh \& Chater (1997) \\
\hline $\mathrm{J} 2031$ & J1501 whiA:: hyg & N. J. Ryding \\
\hline J1979 & $\mathrm{J} 1508 \operatorname{sigF}:: t s r$ & Potúčková et al. (1995) \\
\hline $\mathrm{J} 2210$ & M145 whiH:: hyg & Ryding et al. (1998) \\
\hline $\mathrm{J} 2400$ & M145 whiG:: hyg & This paper \\
\hline $\mathrm{J} 2401$ & M145 whiA:: hyg & This paper \\
\hline $\mathrm{J} 2402$ & M145 whiB::byg & This paper \\
\hline $\mathrm{J} 2404$ & C119 whiG::byg & This paper \\
\hline $\mathrm{J} 2405$ & C119 whiA:: hyg & This paper \\
\hline $\mathrm{J} 2406$ & C119 whiB: : hyg & This paper \\
\hline $\mathrm{J} 2407$ & C119 sigF : :tsr & This paper \\
\hline $\mathrm{J} 2408$ & M145 $\Delta$ whiH::ermE & This paper \\
\hline $\mathrm{J} 2409$ & $\mathrm{~J} 1501 \Delta w h i H::$ ermE & This paper \\
\hline $\mathrm{J} 2412$ & M145 $\Delta$ whiH::ermE $\Delta$ whiE:: hyg & This paper \\
\hline $\mathrm{J} 2413$ & M145 whiA:: hyg $\Delta$ whiH::byg & This paper \\
\hline $\mathrm{J} 2414$ & M145 whiB::hyg $\Delta$ whiH::hyg & This paper \\
\hline M145 & Prototrophic, $\mathrm{SCP}^{-} \mathrm{SCP}^{-} \mathrm{Pgl}^{+}$ & Hopwood et al. (1985) \\
\hline YU106 & proA1 argA1 redE60 $\Delta$ whiE: : hyg $\mathrm{SCP}^{-} \mathrm{SCP}^{-}$ & Yu \& Hopwood (1995) \\
\hline \multicolumn{3}{|l|}{ Plasmids } \\
\hline pIJ698 & $\operatorname{Hyg}^{\mathrm{R}}$ Thio $^{\mathrm{R}}$ & Kieser \& Melton (1988) \\
\hline pIJ2157 & pIJ698 with $w h i B^{+}$in $5.7 \mathrm{~kb}$ insert & Davis \& Chater (1992) \\
\hline pIJ6201 & pIJ698 with $w h i H^{+}$in $4 \mathrm{~kb}$ insert & Ryding et al. (1998) \\
\hline pIJ6204 & pIJ698 with $w h i A^{+}$in $7 \mathrm{~kb}$ insert & Ryding (1995) \\
\hline pIJ6301 & pIJ698 with $w h i G^{+}$in $2 \mathrm{~kb}$ insert & N. J. Ryding \\
\hline
\end{tabular}

using the Kirby-mix procedure of Hopwood et al. (1985). The structures of the $w h i$ gene disruption alleles are summarized in Fig. 1, with the exception of the $\Delta w h i E:: h y g$ allele in which ORFs I-VII of the whiE gene cluster are replaced by the hyg marker (Yu \& Hopwood, 1995). Protoplasts of recipient strains were transformed with around $2 \mu \mathrm{g}$ alkali-denatured chromosomal DNA from donor strains as described previously (Oh \& Chater, 1997). After protoplast regeneration, the antibiotic resistance markers used to disrupt the genes were selected. The presence of disruptant alleles and absence of the corresponding wild-type alleles were verified by PCR or Southern blotting, and the absence of other unsought morphological mutations was confirmed in complementation tests with the corresponding gene on the low-copy-number SCP2derived pIJ698 vector (Table 1).

Microscopical methods. For scanning electron microscopy, colonies were mounted on the surface of an aluminium stub with O. C. T. compound (Agar Scientific) as the mounting medium. The stub was then plunged into liquid nitrogen slush at approximately $-210{ }^{\circ} \mathrm{C}$ to cryopreserve the material. The sample was then transferred onto the cryostage of a CT1500HF cryo-transfer system (Oxford Instruments) attached to a Philips XL30 FEG scanning electron microscope (Philips Electron Optics). Sublimation of surface frost was performed at $-95^{\circ} \mathrm{C}$ for $3 \mathrm{~min}$ before sputter-coating the sample with platinum for $2 \mathrm{~min}$ at $10 \mathrm{~mA}$, at colder than $-110^{\circ} \mathrm{C}$. After sputter-coating, the sample was moved onto the cryo-stage in the main chamber of the microscope, held at approximately $-140{ }^{\circ} \mathrm{C}$. The sample was viewed at $3 \mathrm{kV}$ and photographs were taken using Ilford FP4 120 roll film.

Cultures for light and fluorescence microscopy were set up by inserting a sterile coverslip at a $45^{\circ}$ angle into MM agar and inoculating in the acute angle along the glass surface (Chater, 1972). Coverslips were removed after 2-4 d incubation at $30{ }^{\circ} \mathrm{C}$ and cells on the coverslip surface were either stained with 4',6-diamidino-2-phenylindole (DAPI) for nucleoids (Kwak \& Kendrick, 1996), or stained for the cell wall, with fluorescein-conjugated wheat germ agglutinin (Fluo-WGA) after fixation, treatment with lysozyme and blocking with BSA (Schwedock et al., 1997). To stain DNA in the Fluo-WGAtreated material, 7-aminoactinomycin D (7-AAD) was included at a final concentration of $10 \mu \mathrm{g} \mathrm{ml}^{-1}$ (both fluorescent reagents were obtained from Molecular Probes). The samples were then washed five times with PBS (Sambrook et al., 1989), and mounted for microscopy in PBS containing $50 \%$ glycerol. Samples were studied and photographed using a Zeiss Axiophot microscope equipped for phase-contrast and epifluorescence microscopy. No filters were used for observation of DAPI staining, Zeiss filter set 09 with an added filter cutting off at $560 \mathrm{~nm}$ was used for Fluo-WGA, and filter set 15 was used for 7-AAD. Photographs were taken with Kodak Technical pan film; exposure was at 25 ASA with normal development for phase-contrast illumination, and at 100 ASA with development in HC-110, dilution D, $6 \mathrm{~min}$, for fluor- 


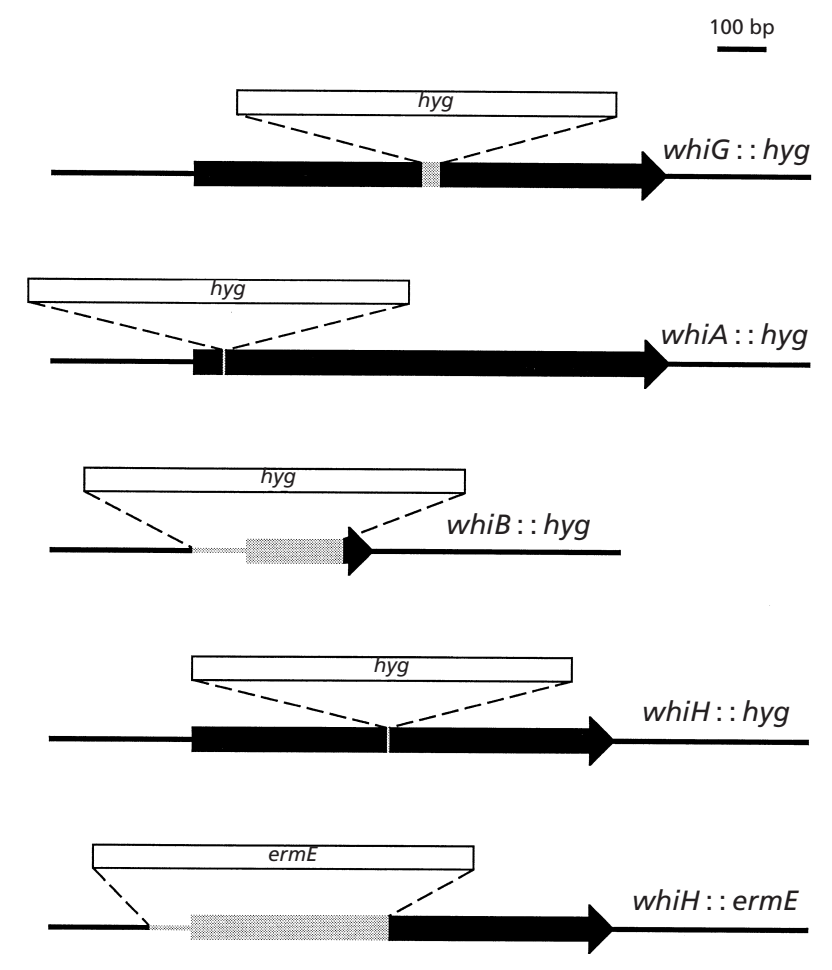

Fig. 1. whi gene disruptions used in this study. The whiG::hyg allele has a 13 codon BamHI segment of whiG replaced by the hyg cassette (Chater et al., 1989). The whiA::hyg allele has the hyg cassette inserted in a Pstl site after the first 21 codons of whiA (N. J. Ryding, N. Hartley, C. J. Bruton, J. A. Aínsa \& K. F. Chater, unpublished). In the whiB::hyg allele, hyg replaces a Bg/ll-Eco47III fragment containing all of whiB (Oh \& Chater, 1997). The whiH::hyg allele has hyg inserted in a BamHI site in the middle of the whiH coding region (Ryding et al., 1998). The $\Delta$ whiH::ermE allele is described in this paper. For references, see Table 1.

escence illumination. Negative images were scanned, processed using Adobe Photoshop software and assigned monochromatic red, green or blue colours to resemble the samples as observed in the microscope.

\section{RESULTS}

Aerial hyphal morphology and septation patterns in whiG, whiA, whiB and whiH disruption mutants: whiH mutants attain some morphological features associated with later stages of sporulation

A series of strains with defined disruptions of the early sporulation regulatory genes $w h i G$, whiA, whiB and whiH were constructed in an isogenic background by transforming the prototrophic, plasmid-free S. coelicolor A3(2) strain M145 with chromosomal DNA from existing disruption mutants. To compare the effects of these mutations on morphological differentiation, colonies that had developed on MM for $5 \mathrm{~d}$ were analysed by scanning electron microscopy (Fig. 2). As is typical for S. coelicolor wild-type strains, the parent M145 colonies were covered with abundant spore chains, which were straight or loosely coiled and often contained several tens of spores (Fig. 2a). None of the mutants formed spore chains. The whiG: hyg mutant J2400 formed long straight aerial hyphae (Fig. 2b), very similar to those observed in previously characterized whiG point mutants (Chater, 1972). Slight constrictions indicating septa were frequently visible. Branches were rare. The whiA:: hyg and whiB::hyg strains J2401 and J2402 closely resembled each other in possessing tightly coiled aerial hyphae (Fig. 2c-d). The coiled regions appeared to lack septal constrictions and branches, and were often very long (see below). The excessive length was further reflected in the scarcity of hyphal tips in fields of J2401 and J2402 compared to those of the other mutants. Similar coils were observed by phase-contrast microscopy of most of the originally isolated whiA and whiB mutants, although one whiA mutant showed considerable fragmentation (Chater, 1972). Finally, and in agreement with previous results from optical microscopy (Chater, 1972; Ryding et al., 1998), the whiH: : hyg strain $\mathrm{J} 2210$ produced loosely coiled aerial hyphae with occasional deep constrictions (Fig. 1e-f).

The patterns of septation in aerial hyphae that had been allowed to develop the mutation-specific characteristic terminal phenotypes were analysed by staining lysozyme-treated cell walls with Fluo-WGA, which binds to oligomers of peptidoglycan. DNA was stained with 7 AAD. Fig. 3 shows a typical developing spore chain of the wild-type strain M145. As described previously for another morphologically wild-type strain, J1508 (Schwedock et al., 1997), ladders of sporulation septa formed synchronously, reaching right up to the tip of the hypha, and single nucleoids were partitioned into the spore compartments. In the phase-contrast microscope, the spore compartments appeared darker than other hyphae and contrasted with the stalk leading up to the spore chains. This difference became more accentuated as sporulation progressed. As exemplified by the piece of a more mature spore chain in the top righthand corner of Fig. 3(a-c), the walls of maturing spores lose susceptibility to staining with Fluo-WGA (Schwedock et al., 1997).

The straight aerial hyphae of $\mathrm{J} 2400$ (whiG) contained crosswalls with a mean spacing of $15 \mu \mathrm{m}$ (Fig. 4a-b). These septa did not lead to cell separation and appeared more similar to vegetative crosswalls than sporulation septa. The presence of such vegetative-like septa correlates well with the shallow constrictions of the aerial hyphal walls seen in the scanning electron microscope (Fig. 2b). The tightly coiled regions of aerial hyphae in J2401 (whiA) were virtually devoid of septa, as shown by the absence of Fluo-WGA-staining crosswalls, and by the continuity of the 7-AAD-staining DNA (Fig. 5a-c). A very similar pattern was seen in $\mathrm{J} 2402$ (whiB). Although septa were usually absent from the coiled regions of the whiA and whiB mutants, rare single septa could be found (Fig. 5b-c). Many coils had 10-20 or even more turns without septa. Given the mean circumference of the coil of $6 \cdot 2 \pm 0 \cdot 5 \mu \mathrm{m}$ (measured for both mutants in scanning electron micrographs like those in Fig. 2c-d), these coiled aseptate regions were far longer than the hyphal compartments delimited by septa 

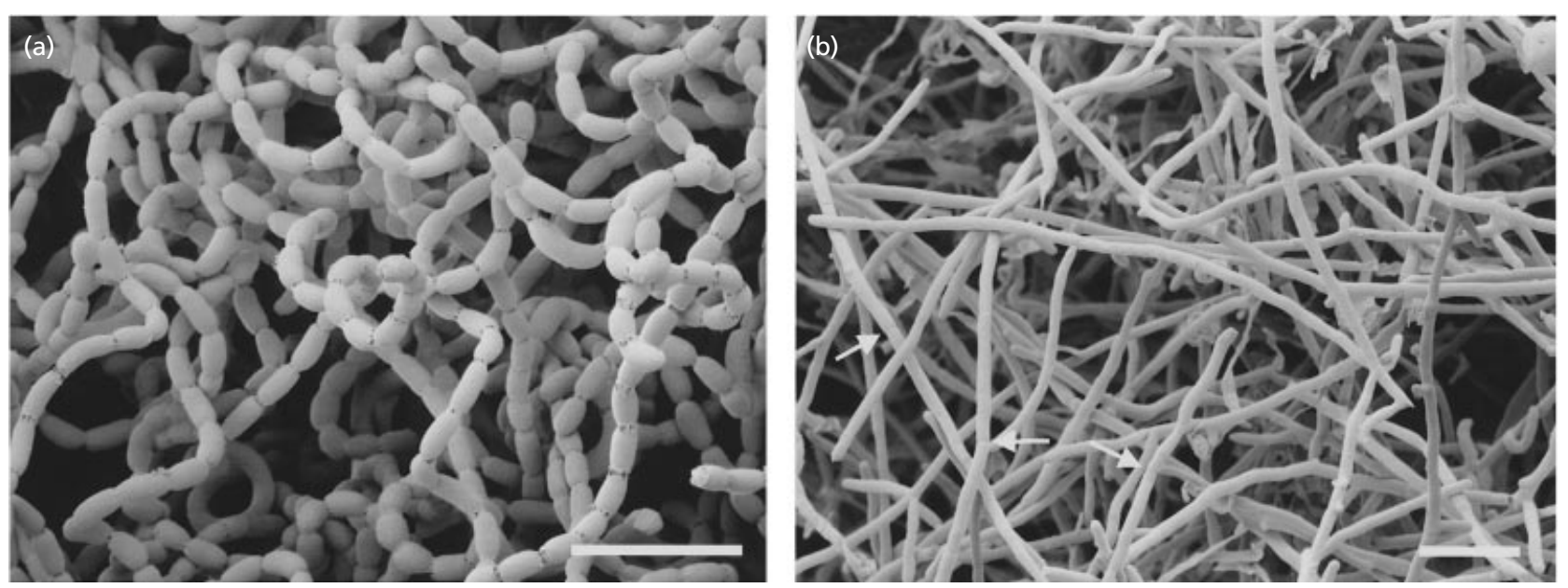

M145

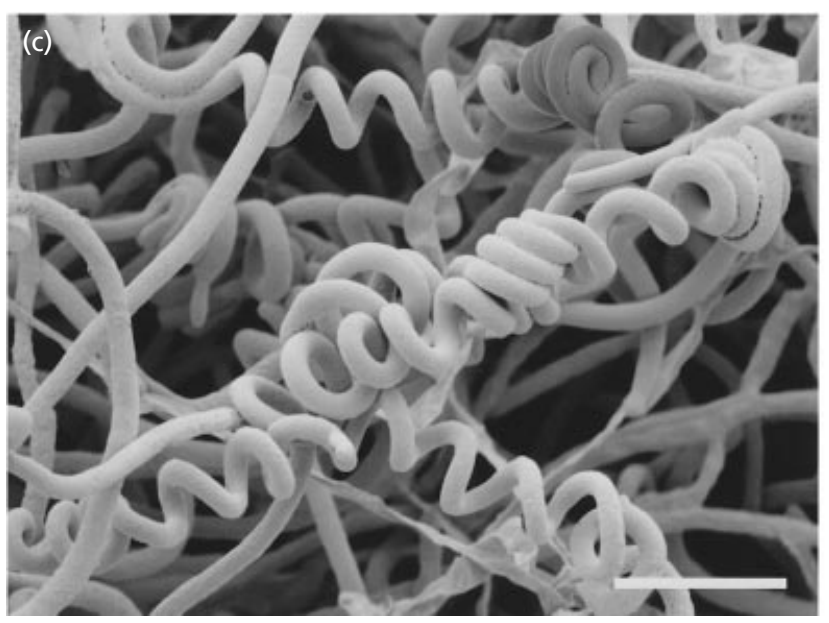

whiA: : hyg

whiG:: hyg

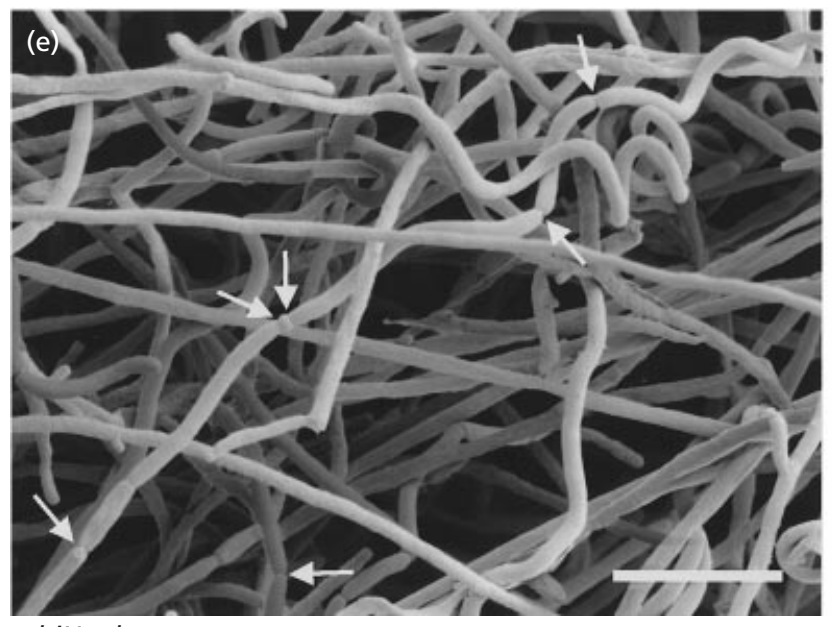

whiH: : hyg

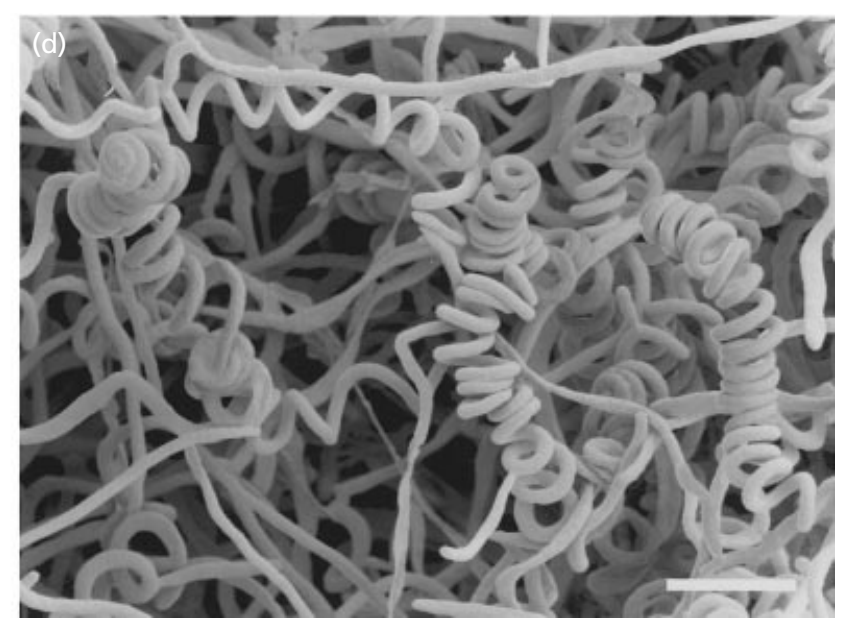

whiB : : hyg

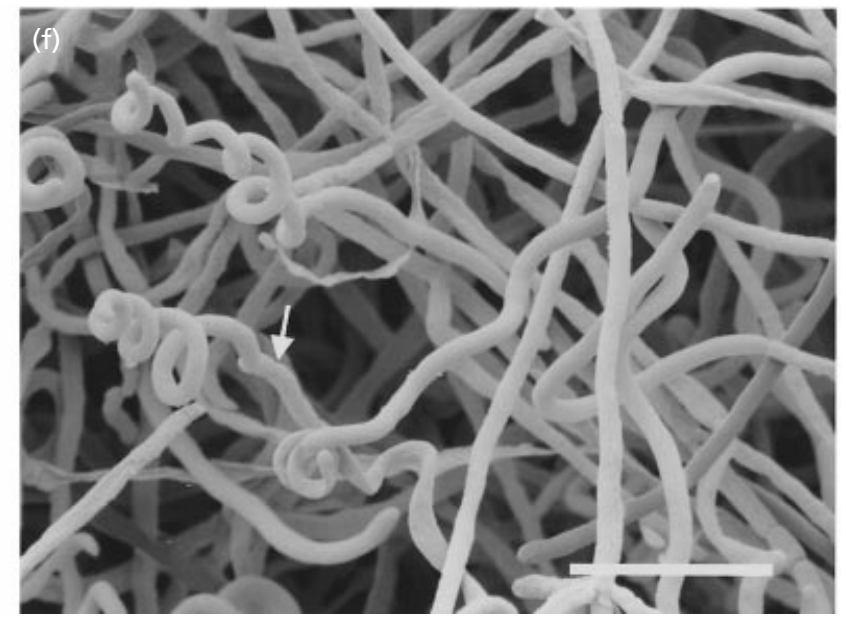

whiH: : hyg

Fig. 2. Scanning electron micrographs of the surface of colonies of the wild-type strain M145 (a) and its whi mutant derivatives J2400 (whiG) (b), J2401 (whiA) (c), J2402 (whiB) (d) and J2210 (whiH) (e-f). Colonies had developed for 5 d on mannitol MM plates before being prepared for electron microscopy. Indentations indicating septation are indicated by arrows. Bars, $10 \mu \mathrm{m}$. 

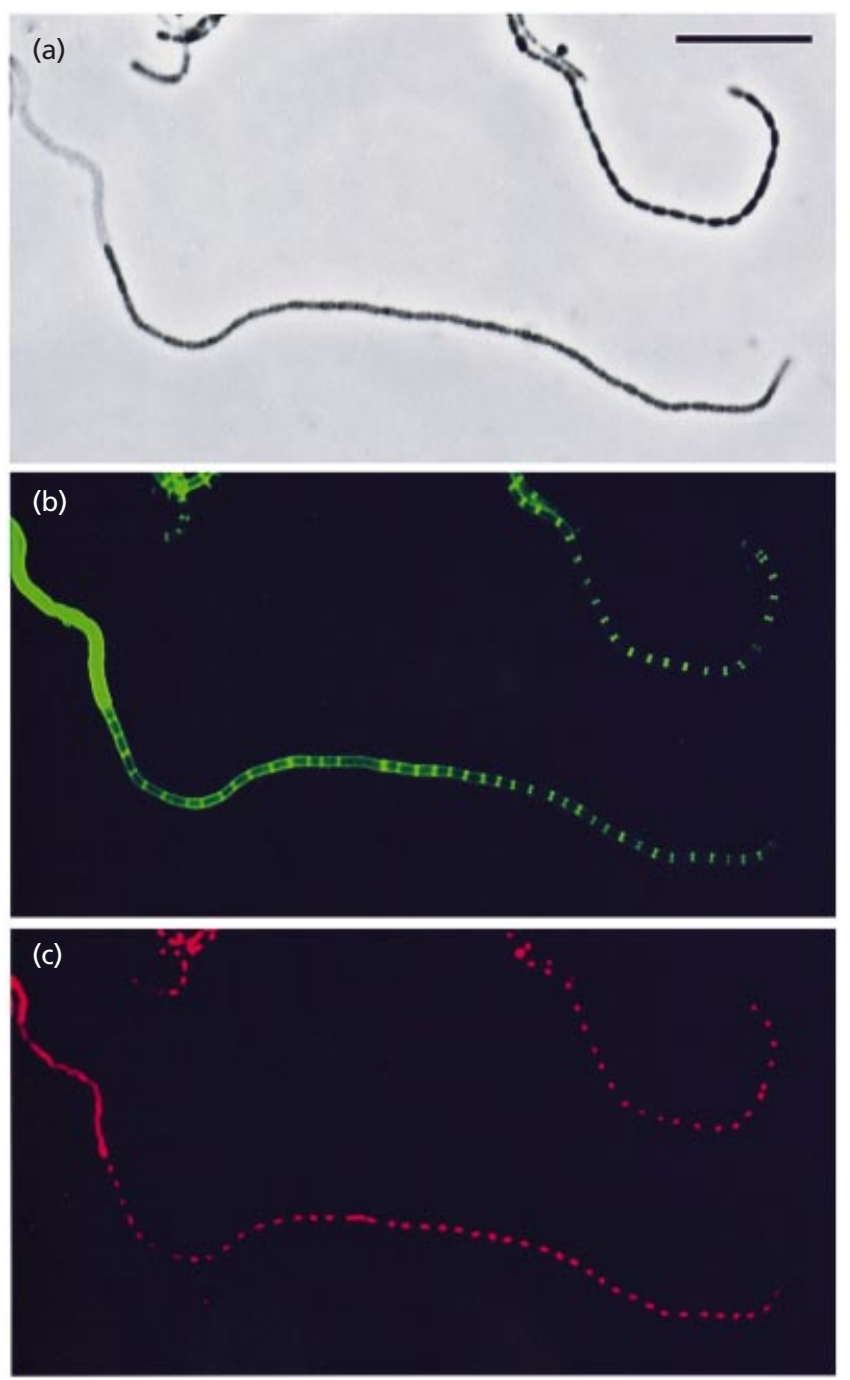

Fig. 3. Characteristic aerial hyphae of the wild-type strain M145. (a) Phase-contrast micrograph; (b, c) fluorescence micrographs of hyphae stained with Fluo-WGA (b) and 7-AAD (c). The figure shows representative aerial hyphae from colonies that had developed for $3 \mathrm{~d}$ on mannitol MM plates before being prepared for microscopy. Bar, $10 \mu \mathrm{m}$.

in the whiG mutant J2400. Such lengths would correspond to chains of around 100 or more spores, which are rarely seen in the M145 strain, even when looking at young Fluo-WGA-stained aerial hyphae where septa can be counted before the spore chains start to break up (data not shown).

Unlike developing spore chains of the wild-type, aerial hyphae of $\mathrm{J} 2400$ (whiG), J2401 (whiA), or J2402 (whiB) did not become dark in phase-contrast illumination, nor did they fail to stain with Fluo-WGA. In contrast, the loosely coiled aerial hyphae of whiH mutants were often fragmented into segments of different lengths, the fragments appeared dark in phase-contrast and their cell walls stained very poorly or not at all with Fluo-WGA. This was first observed with strain J2210 (whiH::hyg; data not shown), and subsequently with the
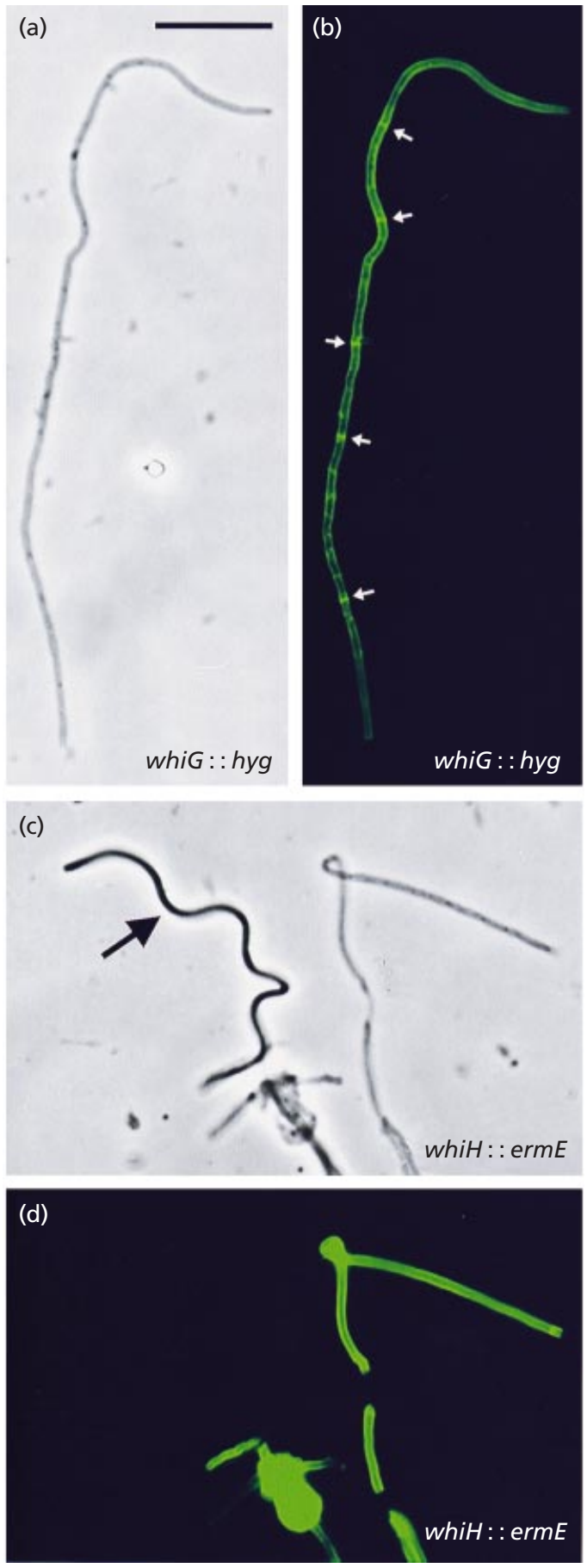

Fig. 4. Characteristic aerial hyphae of whiG and whiH disruption mutants. Phase-contrast $(a, c)$ and fluorescence (FluoWGA) (b, d) micrographs of $\mathrm{J} 2400$ (whiG) (a, b) and $\mathrm{J} 2408$ (whiH) (c, d) show representative aerial hyphae from colonies that had developed for $3 \mathrm{~d}$ on mannitol MM plates before being prepared for microscopy. The small arrows in (b) indicate the widely spaced crosswalls found in whiG mutant aerial hyphae (other, more faintly staining positions of fluorescence did not correspond to crosswalls when viewed in different focal planes). The arrow in (c) indicates a hyphal fragment of the kind referred to in the text as phase-dark. Bar, $10 \mu \mathrm{m}$. 


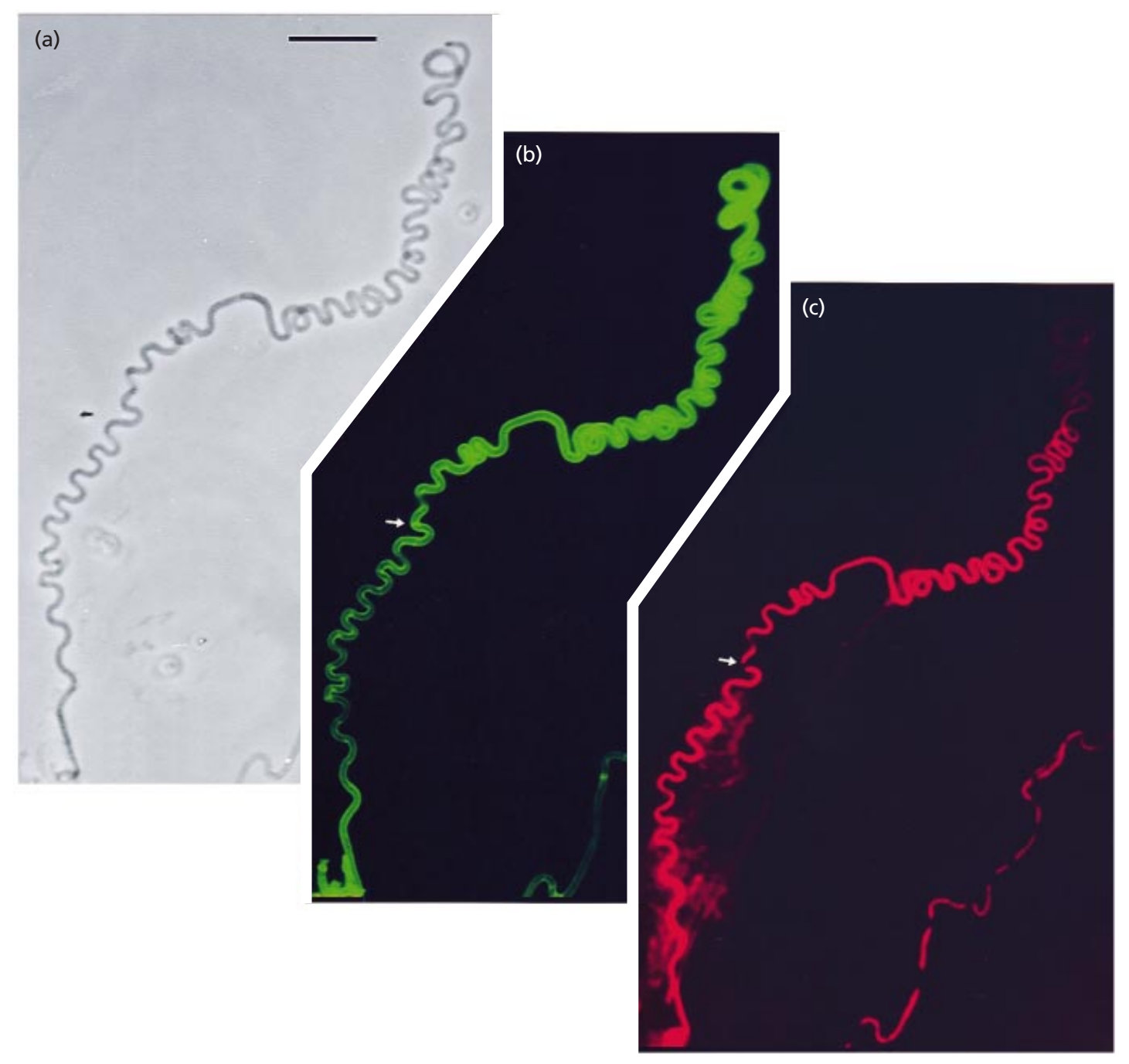

Fig. 5. Characteristic aerial hyphae of strain J2401 (whiA::hyg). Phase-contrast micrograph (a), and fluorescence micrographs of hyphae stained with Fluo-WGA (b) and 7-AAD (c). The panels show a representative coiled aerial hypha from a colony that had developed for $3 \mathrm{~d}$ on mannitol MM plates before being prepared for microscopy. Arrows point to a probable septum. The results with $\mathrm{J} 2402$ (whiB::hyg) were indistinguishable from those shown here for J2401 (whiA::hyg). Bar, $10 \mu \mathrm{m}$.

$\Delta$ whiH::ermE null mutant J2408 (Fig. 4c-d), the construction of which is described below. The fragmentation of aerial hyphae of whiH mutants, and the deep constrictions observed in electron micrographs of such hyphae, suggested that a few sporulation septa had formed, which was in stark contrast to the other whi mutants analysed here.

\section{A whiH null mutant produces perceptible amounts of the whiE-encoded grey spore pigment}

The production of aerial hyphal fragments with sporelike characteristics in the whiH::hyg strain J2210 indicated that whiH may not be required for all late sporulation processes. This was further supported by the previous observations that whiH mutants develop a slightly grey aerial mycelium while whiG, whiA and
whiB colonies remain pure white (Ryding et al., 1998) and by low, but detectable, transcription of the major operon of whiE spore pigment genes and of sigF, encoding a late sporulation $\sigma$ factor, in a whiH point mutant (Kelemen et al., 1996, 1998). However, the possibility could not be excluded that previously studied whiH mutants retained residual whiH activity because they contained either missense mutations, a frameshift in the C-terminal half, or uncharacterized mutations (Chater, 1972; Ryding et al., 1998). Also, the whiH : : hyg insertion mutation, which gives rise to a very low level of grey pigmentation, allows expression of the $\mathrm{N}$ terminal half of WhiH, including a putative DNAbinding motif (Ryding et al., 1998). A knockout mutation of $w h i H$ was therefore constructed by deleting the promoter and the first half of the gene and replacing it with the ermE gene. When introduced into M145 to 


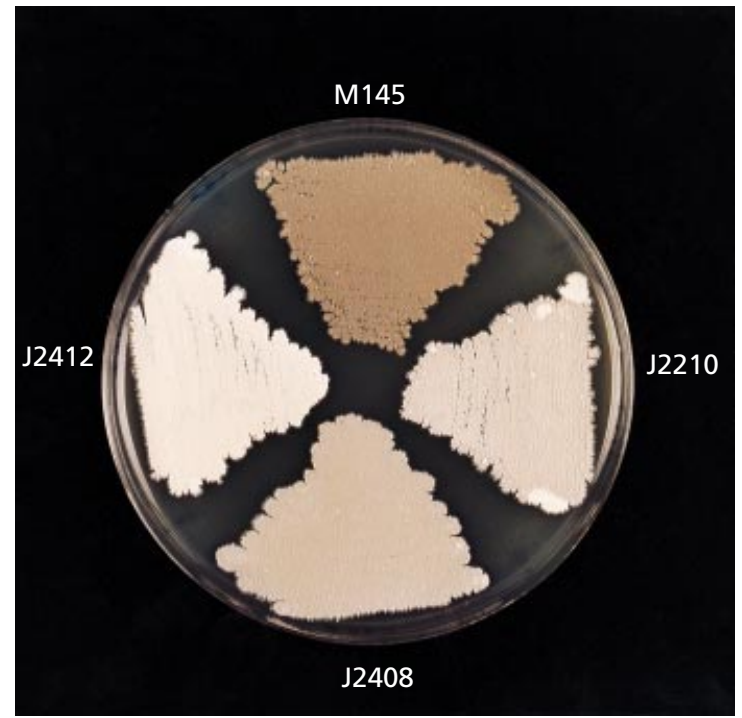

Fig. 6. Pigmentation of aerial mycelium on the surface of strains M145, J2210, J2408 and J2412 (mannitol MM, 4 d). J2412 (a whiE whiH::ermE double mutant) is creamy white whereas the whiH::ermE single mutant (J2408) is almost as grey as the wild-type M145. The whiH::hyg mutant (J2210), which retains the whiH promoter and much of the whiH gene, is pale grey.

produce strain J2408, this $\Delta$ whiH::ermE allele gave aerial hyphae which were morphologically and cytologically very similar to those of other whiH mutants (see Fig. 4c-d and below). The aerial mycelium of J2408 colonies accumulated readily perceptible grey pigment, to a level intermediate between J2210 (whiH::hyg) and M145 (Fig. 6). The extent of grey pigmentation was medium-dependent (e.g. J2408 appears white on soya flour mannitol agar), which may explain why whiH mutants had appeared no less white than any other white colony mutants in the original screen (Hopwood et al., 1970; see also Ryding et al., 1998).

The grey pigment of the wild-type is of polyketide origin, and its synthesis is directed by the whiE gene cluster (Davis \& Chater, 1990; Yu \& Hopwood, 1995). To find out whether the grey pigment of whiH mutants was the same as that of the wild-type, the whiE::hyg mutation of Yu \& Hopwood (1995) was introduced into J2408. The resulting strain, J2412, lacked the grey pigment and had, like other whiE mutants, a very pale cream-coloured aerial mycelium (Fig. 6), but retained all other morphological characteristics of whiH mutants (data not shown). We concluded that the greyness of whiH mutants is indeed due to the whiE-encoded spore pigment, and therefore that whiE expression is not completely dependent on whiH. This result was consistent with the previous finding of a low level of whiE mRNA in a whiH point mutant (Kelemen et al., 1998).

\section{Condensation and aberrant partitioning of nucleoids in aerial hyphae of whiH mutants}

Spore formation involves accurate segregation of one nucleoid to each prespore compartment (although oc- casional abnormal events occur, as is evident from Fig. $3 a-c)$. We used DAPI staining of methanol-fixed aerial hyphae to examine how nucleoid partitioning was affected by the whi mutations. As reported for several Streptomyces species (Kwak \& Kendrick, 1996; Miguélez et al., 1998; Schwedock et al., 1997), separation of nucleoids is not seen in the wild-type strains until septa have started forming. Thereafter, nucleoids stain more intensely and become smaller (Fig. 7a-b). No such condensation was seen in $\mathrm{J} 2400$ (whiG), J2401 (whiA) and $\mathrm{J} 2402$ (whiB), nuclear material remaining more or less continuous throughout the straight or coiled aerial hyphae (Fig. 7c-e). However, away from apical parts, in particular in J2400, and in older parts of hyphae, the staining was more patchy and unevenly distributed (data not shown). Results obtained with 7-AAD staining of paraformaldehyde/glutaraldehydefixed mutant aerial hyphae agreed with the results from DAPI staining (data not shown). In contrast, the whiH mutant J2210 displayed a distinctly different pattern of nucleoid staining. In the phase-dark and often loosely coiled fragments that formed in many aerial branches, nucleoids were partitioned into bodies of variable sizes and distributed unevenly along the length of the fragments, leaving large DNA-free spaces between them (Fig. 7f). There were no nucleoids close to the poles of the phase-dark fragments, and the total length of DNAcontaining sections was about equal to the total length of DNA-free sections. Otherwise, no obvious pattern of distribution could be discerned. The nucleoids fluoresced intensely and appeared to be condensed, contrasting sharply with the non-condensed DNA in the hyphal stems leading up to the phase-dark fragments. Often, the stems appeared to be lysed in ageing material, while the phase-dark fragments persisted. Further analysis revealed this pattern of DNA staining in all of 13 independent $w$ hiH mutants obtained after UV or NTG (nitrosoguanidine) mutagenesis (listed in Methods section; representative examples shown in Fig. 7 g-j).

A previous report indicated that $\operatorname{sig} F$, encoding a sigma factor required for some late stages of sporulation, may be needed for condensation of DNA in mature spores (Potúcková et al., 1995). To determine whether sigF influenced the whiH phenotype, a sigF::tsr whiH119 double mutant, J2407, was constructed. It lost the grey pigmentation of the parent strain C119 (as expected, since a major whiE promoter is unexpressed in sigF mutants; Kelemen et al., 1998), but the aerial hyphal morphology and DNA condensation and segregation pattern were indistinguishable from C119 (data not shown). We have no explanation for the disparity between our data showing sigF-independent DNA condensation in the whiH mutant, and the earlier results of Potúčková et al. (1995) obtained with a $w h i H^{+}$strain.

\section{whiG, whiA and whiB are epistatic to whiH in morphological studies of double mutants}

Since $w h i H$ was not absolutely required for parts of the wild-type sporulation processes, while whiG, whiA and $w h i B$ mutants were completely blocked at early stages of 

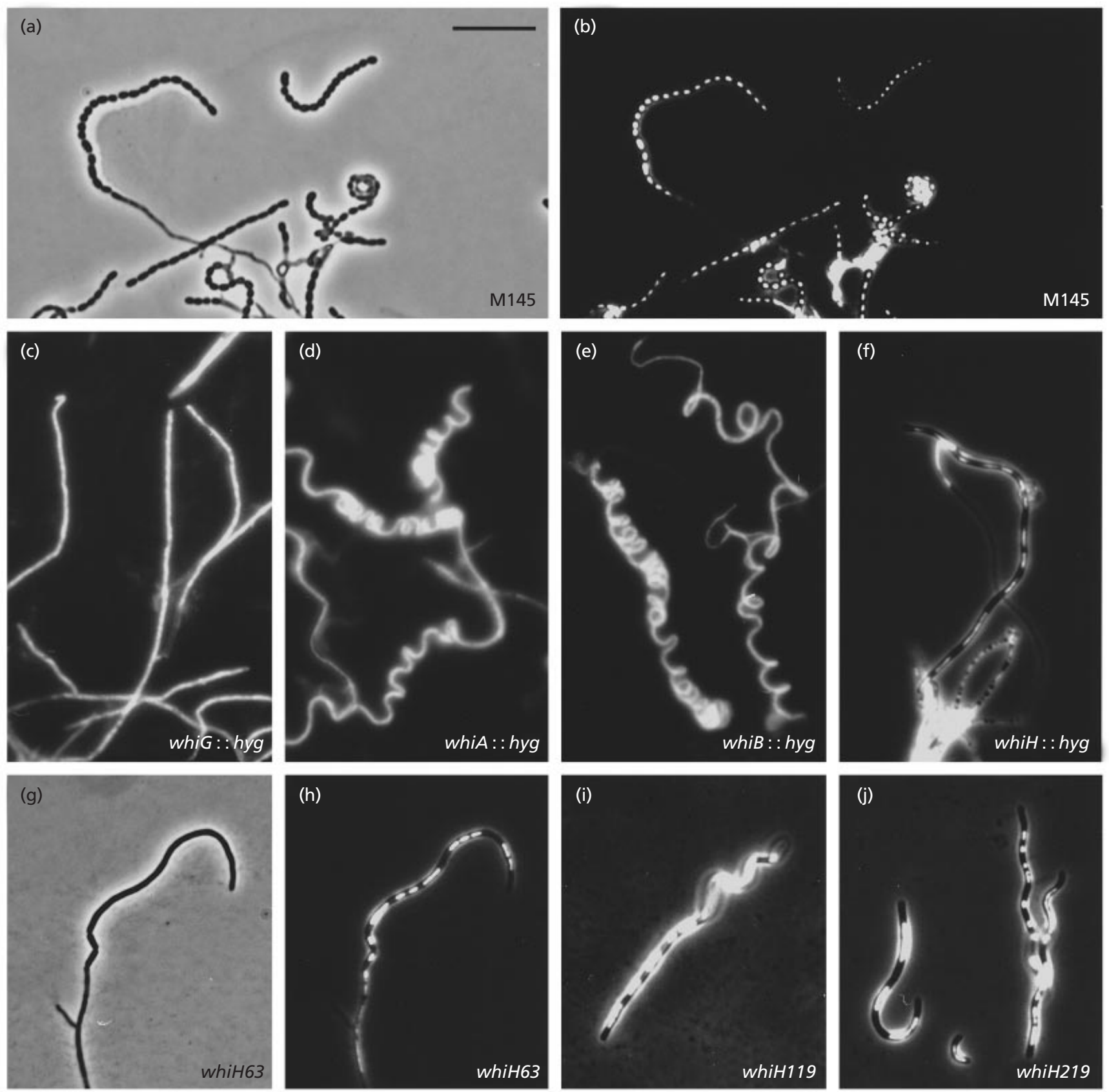

Fig. 7. DAPI staining of characteristic aerial hyphae of the wild-type strain M145 (b), J2400 (whiG::hyg) (c), J2401 (whiA::hyg) (d), J2402 (whiB::hyg) (e), J2210 (whiH::hyg) (f), C63 (whiH63) (h), C119 (whiH119) (i) and C219 (whiH219) (j). Phase-contrast micrographs of strains M145 (a) and C63 (g) are also shown. Bar, $10 \mu \mathrm{m}$.

sporulation, whiG, whiA and whiB mutations would be expected to be epistatic to whiH mutations. However, early phase-contrast microscopic studies of double mutants had indicated that the epistasis pattern was $G>H>A, B$ (Chater, 1975). Those results were based on highly non-isogenic strains arising from genetic crosses. Exploiting our ability to construct strains in an isogenic background, and using the easily scorable DAPI staining pattern, the epistasis was re-examined. The
whiG:: hyg, whiA:: hyg and whiB::hyg mutations were transferred to strain C119, which contains a point mutation (whiH119) causing an amino acid substitution near the supposed WhiH DNA-binding domain, and has a phenotype like that of the whiH null mutant (Ryding et al., 1998). Derivatives of the double mutants containing functional alleles of the relevant whi genes on low-copy-number plasmids were also studied as controls expected to show the relevant single mutant phenotypes. 

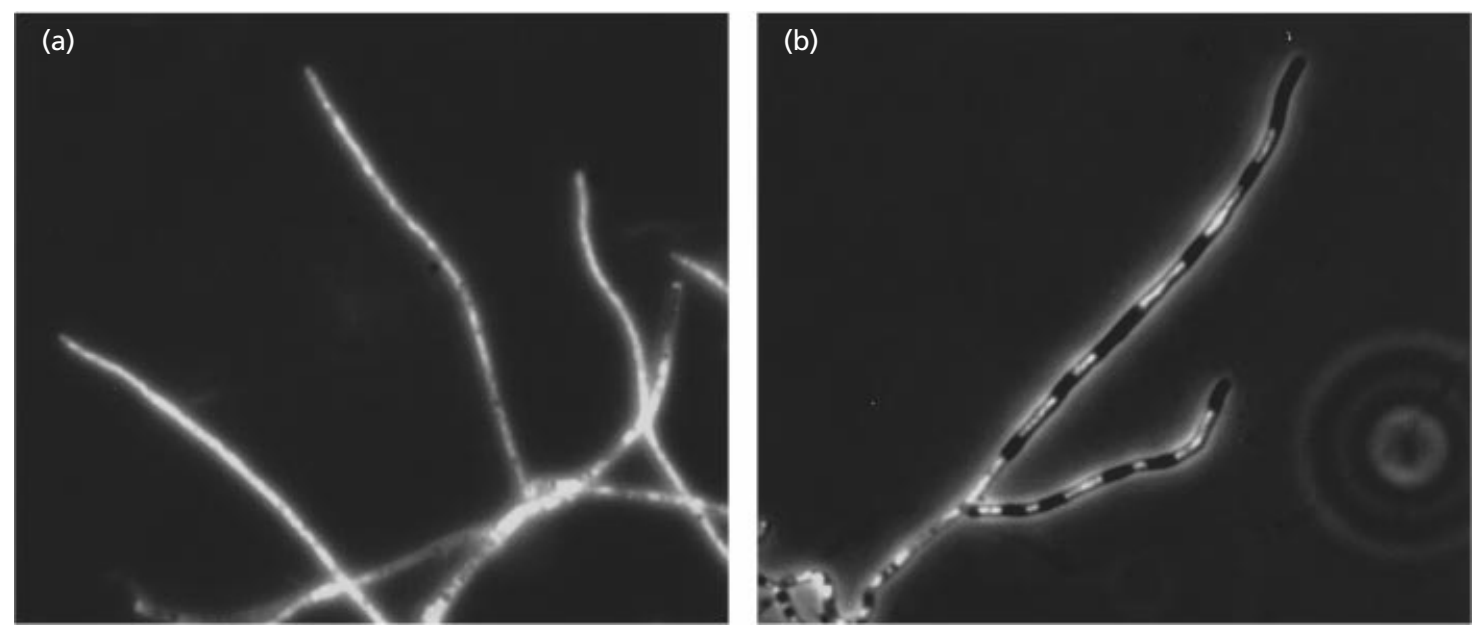

whiG: : hyg whiH119

whiG: : hyg whiH119+ whiG ${ }^{+}$plasmid
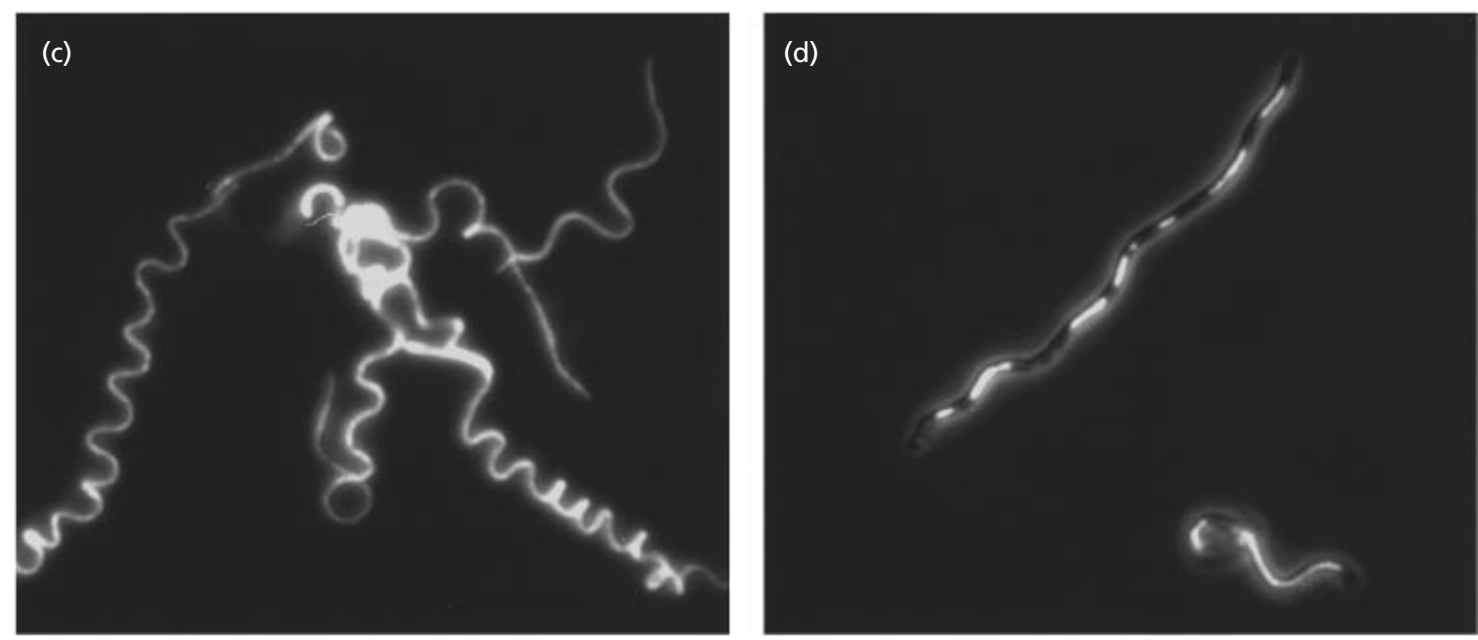

whiA:: hyg whiH119

whiA: : hyg whiH119+ whiA ${ }^{+}$plasmid
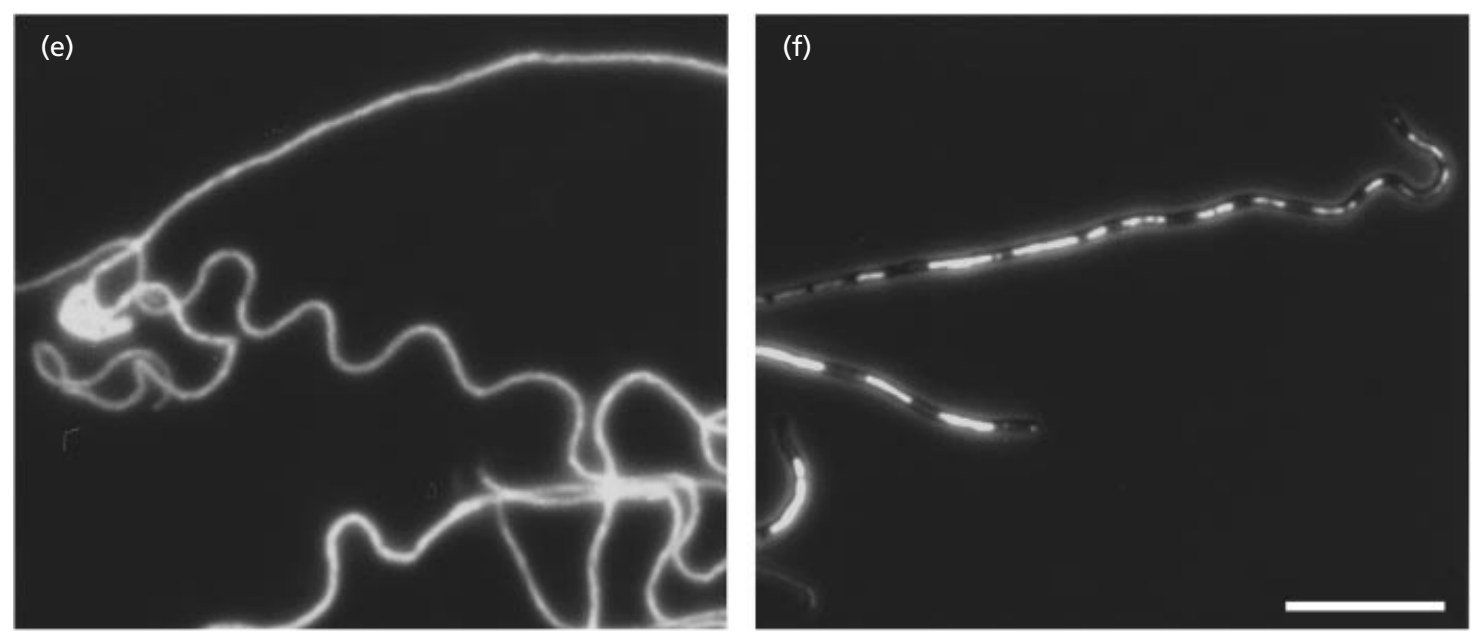

whiB:: hyg whiH119

whiB: : hyg whiH119+ whiB ${ }^{+}$plasmid

Fig. 8. DAPI staining of aerial hyphae of the double whi mutants J2404 (whiG::hyg whiH119) (a), J2405 (whiA::hyg whiH119) (c), and J2406 (whiB:: hyg whiH119) (e), and of the same strains where the whiG::hyg had been complemented by whiG on plasmid plJ6301 (b), whiA::hyg had been complemented by whiA on plJ6204 (d) and whiB::hyg had been complemented by whiB on plJ2157 (f), respectively. Bar, $10 \mu \mathrm{m}$. 
Table 2. Phenotypes of constructed whiA whiH and whiB whiH double mutants in the presence and absence of plasmid-borne functional alleles

\begin{tabular}{|c|c|c|c|}
\hline Strain & Plasmid & $\begin{array}{c}\text { Colour of aerial } \\
\text { mycelium* }\end{array}$ & Morphology of aerial hyphae \\
\hline M145 & - & Dark grey & Sporulation \\
\hline $\mathrm{J} 2408(w h i H)$ & - & Pale grey & Short, phase-dark fragments \\
\hline $\mathrm{J} 2413$ (whiH whiA) & $\begin{array}{l}- \\
\text { pIJ698 (vector) } \\
\text { pIJ6201 }\left(w h i H^{+}\right) \\
\text {pIJ6204 }\left(w h i A^{+}\right)\end{array}$ & $\begin{array}{l}\text { White } \\
\text { White } \\
\text { White } \\
\text { Very pale grey }\end{array}$ & $\begin{array}{l}\text { Long, loose coils } \\
\text { Long, straight hyphae or very loose coils } \\
\text { Long, tight coils } \\
\text { Short, phase-dark fragments }\end{array}$ \\
\hline $\mathrm{J} 2414($ whiH whiB) & $\begin{array}{l}- \\
\text { pIJ698 (vector) } \\
\text { pIJ6201 }\left(w h i H^{+}\right) \\
\text {pIJ2157 }\left(w h i B^{+}\right)\end{array}$ & $\begin{array}{l}\text { White } \\
\text { White } \\
\text { White } \\
\text { Very pale grey }\end{array}$ & $\begin{array}{l}\text { Long, loose coils } \\
\text { Long, straight hyphae or very loose coils } \\
\text { Long, tight coils } \\
\text { Short, phase-dark fragments }\end{array}$ \\
\hline
\end{tabular}

*Evaluated after $5 \mathrm{~d}$ growth on mannitol MM medium.

†Examined by phase-contrast microscopy after $4 \mathrm{~d}$ growth on mannitol MM medium.

In agreement with previous epistasis data, whiG was epistatic to whiH: the aerial hyphae of strain J2404 (whiG whiH) were indistinguishable in morphology and DNA staining pattern from those of $\mathrm{J} 2400$ (whiG) (Fig. 8a). The phenotype of the double mutant was not affected by introduction of $w h \mathrm{iH}^{+}$on pIJ6201 (not shown), but the complementation of whiG by introducing pIJ6301 gave a characteristic whiH phenotype (Fig. 8b). J2405 (whiA whiH) was similar to the whiA single mutant, although the coils were slightly less tight (Fig. 8c). The whiB whiH strain J2406 had a clearly reduced tightness of the coils in comparison to the whiB single mutants (Fig. 8e). However, the coils of the whiA whiH and whiB whiH mutants were very long, appeared non-septated and had continuously dispersed nuclear material, in this respect closely resembling the whiA and whiB single mutants. Complementation of whiA::hyg in J2405 by pIJ6204, and of whiB: :hyg in J2406 by pIJ2157, restored the $w$ hiH phenotypes completely (Fig. $8 \mathrm{~d}, \mathrm{f}$ ), while introduction into either strain of the $w h \mathrm{hH}^{+}$ plasmid pIJ6201 only increased the tightness of the coils (data not shown). In summary, whiG, whiA and whiB mutations were epistatic to whiH119 on the criteria that they completely abolished the formation of phase-dark hyphal fragments, the occasional sporulation-like septation, and the separation and condensation of nucleoids that occurred in the whiH119 single mutant. In addition, they abolished the pale grey pigmentation of C119 and gave pure white aerial mycelium (data not shown).

The only detectable effect of whiH119 when it was combined with whiA or whiB disruptions was the decreased degree of coiling of aerial hyphae. To further investigate this and to exclude the possiblity that it was related to any residual activity of the whiH119 allele, double mutants were constructed also in J2408 $(\Delta w h i H:: e r m E)$. Both whiA::hyg and whiB::hyg abolished the grey pigmentation of the J2408 parent and prevented the formation of phase-dark aerial hyphal fragments with condensed and separated nucleoids, thus exactly reproducing the results obtained with the C119 strain (Table 2). Aerial hyphae of both double mutants were long coils which appeared looser than the whiA::byg (J2401) or whiB::hyg (J2402) single mutants. Plasmid-borne functional alleles of $w h i$ genes were introduced into the double mutants. The vector itself (pIJ698) reduced the tightness of the coiling of aerial hyphae further, so that the double mutants containing pIJ698 had mostly straight or slightly wavy aerial hyphae (Table 2). As expected, pIJ6204 $\left(w h i A^{+}\right)$ restored the whiH phenotype to J2413, and pIJ2157 $\left(w h i B^{+}\right)$restored the $w h i H$ phenotype to J2414. The whiH $H^{+}$-containing plasmid pIJ6201 gave rise to tight coiling of aerial hyphae in both J2413 and J2414, reminiscent of the phenotype of whiA or whiB single mutants (Table 2). These results showed that a whiH null allele also caused reduced coiling in whiA or whiB mutants.

\section{DISCUSSION}

By examining the morphology and cytology of a series of whi disruption mutants in an isogenic background, we found that inactivation of whiG, whiA and whiB completely prevented all traces of sporulation septation and later stages of spore chain development, while the whiH phenotype was more complex. In the following, the significance of these results and earlier observations on the mutant phenotypes are discussed. A model for commitment to sporulation is suggested, involving two crucial developmental decision points defined by whiG and $w h i A / w h i B$, respectively (Fig. 9). Additional stages or decision points may possibly be recognized when further $w h i$ genes are analysed in detail. The model also suggests how the characteristic whiG, whiA, whiB and whiH phenotypes relate to the stage at which normal development is blocked in the respective mutant. 


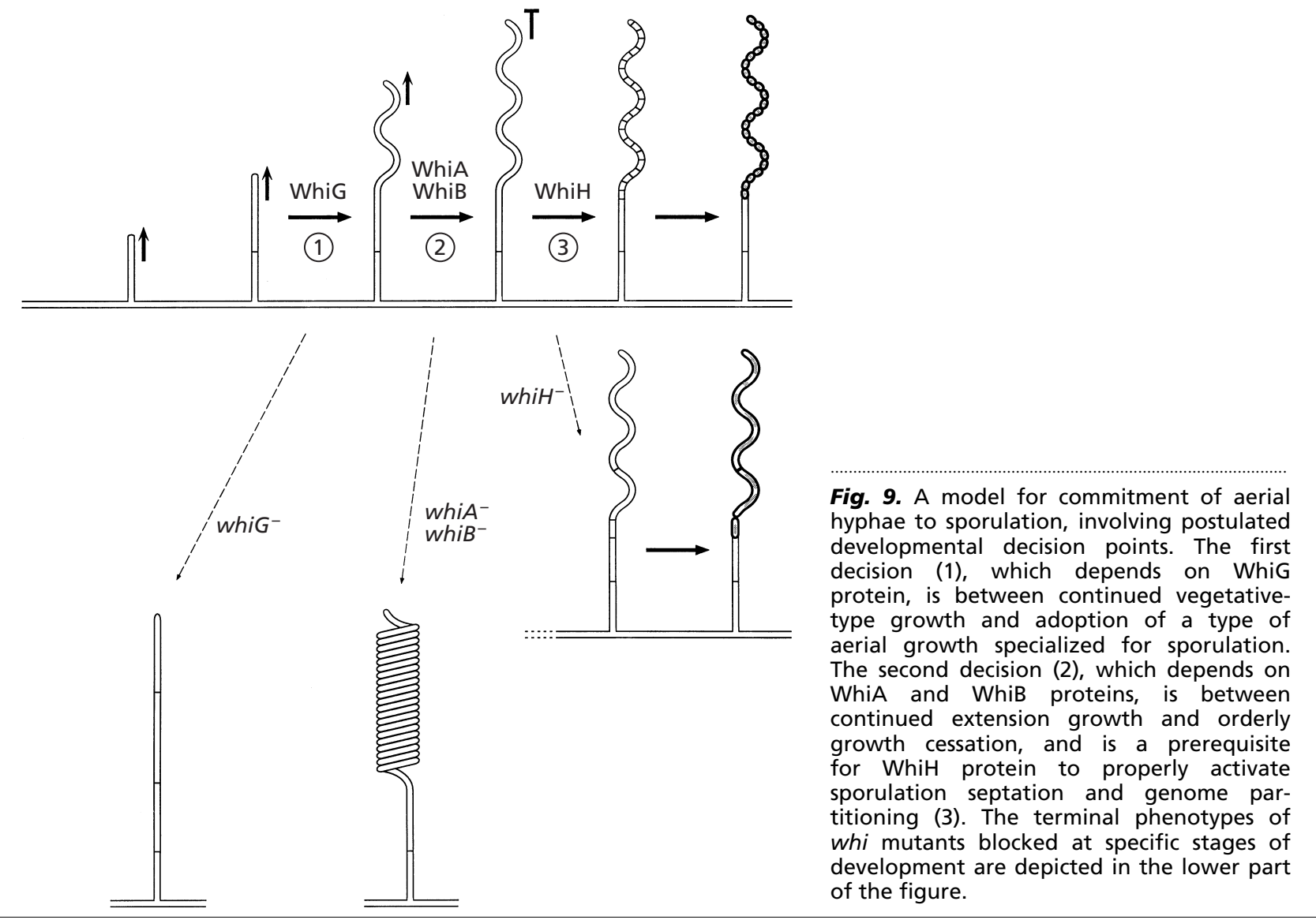

\section{whiG and the primary commitment of aerial hyphae to sporulation}

The aerial hyphae of whiG mutants are straight and septa are laid down with a spacing similar to that reported for vegetative growth of the closely related organism Streptomyces granaticolor (Kretschmer, 1982), in accord with the observation of widely spaced FtsZ rings in whiG aerial hyphae (Schwedock et al., 1997). The structure of the septa in whiG aerial lyphae, as seen by transmission electron microscopy of thin sections, is typical of vegetative septa (Plaskitt \& Chater, 1995). whiG is epistatic to whiA, whiB and whiH (Chater, 1975; this paper), as well as to another whi gene, whil (Chater, 1975), which was not studied here because it was cloned only recently (J. A. Aínsa, H. D. Parry \& K. F. Chater, unpublished). Furthermore, additional copies or increased expression of $w h i G$ cause premature and ectopic sporulation, implying that it is a crucial determinant of the decision to sporulate (Chater et al., 1989; Méndez \& Chater, 1987). Altogether, these observations indicate that there is a whiG-dependent developmental decision point that commits aerial hyphae to sporulation. This decision leads, among other things, to coiling of the apical parts of hyphae, indicative of a switch in the structure of the cell wall (Fig. 9). This could involve a change in the synthesis of new cell wall as it is laid down in the apical region after the whiGdependent switch. Alternatively, it could be accounted for by modification of existing peptidoglycan, an alternative that would raise the further question of how to explain the abrupt discontinuity between straight stem and coiled apex. A continual failure to carry out the whiG-dependent developmental decision would give rise to continued growth of straight and in many respects undifferentiated aerial hyphae (Fig. 9).

The whiG gene product is an RNA polymerase $\sigma$ factor, $\sigma^{\text {Whig }}$ (Chater et al., 1989; Tan et al., 1998), of a subclass whose homologues in other bacteria (e.g. $\sigma^{\text {FliA }}$ in Salmonella typhimurium) are subject to regulation by anti- $\sigma$ factors responsive to morphological checkpoints (Hughes et al., 1993). It has previously been suggested that the morphologically coupled release of $\sigma^{\text {Whig }}$ from an anti- $\sigma$ factor may be critical in initiating sporulation (Losick \& Shapiro, 1993; Kelemen et al., 1996). As one result, $\sigma^{\text {WhiG }}$ RNA polymerase would transcribe a gene or genes influencing cell wall structure. We suggest that the transition from straight to curled growth of a hypha occurs very soon after $\sigma^{\text {WhiG }}$ RNA polymerase has become active, and provides a visible record of the time at which the checkpoint was successfully negotiated. Another whiG-dependent gene encodes a possible glycine-betaine-binding protein, perhaps contributing to turgor for continued growth of the apical compartment after it has been cut off from direct contact with the substrate mycelium (Tan et al., 1998). Indeed, this septation event might itself provide the checkpoint, 
as suggested by Losick \& Shapiro (1993); a consequent transient limitation of nutrients or of turgor might be more direct sources of the $\sigma^{\mathrm{WhiG}}$-activating signal. Further $\sigma^{\text {WhiG }}$ targets include the sporulation regulatory gene whiH (Ryding et al., 1998).

\section{A putative dependence of sporulation septation on growth cessation may provide the basis of a whiA- and whiB-mediated developmental checkpoint}

The whiG-dependent switch in cell wall structure apparently works in whiA and whiB mutants since these develop tightly coiled aerial hyphae. The epistasis of whiG to whiA and whiB is consistent with this conclusion (Chater, 1975). Mutants in whiA or whiB lack sporulation septation or nucleoid segregation and condensation, and develop abnormally long and coiled aerial hyphae. This identifies a putative second developmental decision point required for formation of sporulation septa and for appropriate limitation of aerial growth (Fig. 9). On the other hand, aerial hyphae of whiH mutants are not abnormally long, so they appear to retain proper control of the decision to stop aerial growth.

The fact that whiH mutants make reduced numbers of sporulation septa, yet cease growth properly, indicates that a full commitment to sporulation septation is not a prerequisite for orderly growth cessation. This leads us to suggest tentatively that, instead, growth cessation is a prerequisite for sporulation septation, and that whiA and $w h i B$ affect elongation more directly than septation. Nevertheless, it remains possible that a whiA/whiBdependent initiation of sporulation septation (even if just a few septa are formed as in whiH mutants) could lead to cessation of hyphal elongation.

Properly controlled aerial hyphal cell wall structure, as reflected in the extent of coiling of aerial hyphae, as well as other sporulation events such as sporulation septation, nucleoid condensation, spore pigment synthesis and transcription of $s i g F$ and $w h i E$, all require whiA and whiB (Kelemen et al., 1996, 1998). Although these dependences may conceivably be fairly directly exerted at the level of transcription of the relevant genes, it is quite possible that some or all of the events are more directly affected by the morphological checkpoint in which whiA and whiB are implicated. The sequences of the whiA and whiB genes are known but have not yet provided any unambiguous clues about their molecular function (Davis \& Chater, 1992; N. J. Ryding, J. A. Aínsa, N. Hartley, C. J. Bruton \& K. F. Chater, unpublished).

\section{Some later sporulation processes are initiated in whiH mutants}

Inactivation of whiH does not fully block all aspects of spore chain development. First, all whiH alleles examined to date, including the $\Delta w h i H:$ :ermE null allele, allow synthesis of detectable amounts of a grey spore pigment. We show here that this pigmentation is directed by the whiE gene cluster which is responsible for the grey polyketide pigment of wild-type spores. Consistent with this, very low levels of transcription of the late sporulation loci sigF and whiE were detectable in a whiH point mutant (Kelemen et al., 1996, 1998), whereas no transcription of these loci was detected in whiG, whiA and whiB strains. Second, a few irregularly spaced apparent sporulation septa divide whiH mutant aerial hyphae into shorter fragments, and only a few widely spaced FtsZ rings were reported in whiH::hyg aerial hyphae by Schwedock et al. (1997). Some of these rings may have corresponded to the occasional putative sporulation septa formed in whiH strains. Third, the fragments formed from whiH aerial hyphae become phase-dark and their cell wall becomes resistant to staining with Fluo-WGA in a manner reminiscent of developing spore chains. Fourth, nucleoids in these fragments condense and are partitioned (albeit aberrantly).

We interpret these observations and the epistasis of whiG, whiA and whiB to all these aspects of whiH to mean that the first two developmental decision points do not require whiH (Fig. 9). The whiH mutants initiate later sporulation processes, but execute them incorrectly or only to a limited extent. In the simplest model, whiH would act after $w h i G, w h i A$ and $w h i B$ and be dependent on these genes. Indeed, a direct dependence of whiH transcription on $\sigma^{\text {Whig }}$ has been demonstrated (Ryding et al., 1998). The situation appears, however, to be more complex than this, as whiH mutations clearly reduce the tight coiling that develops in whiA and whiB aerial mycelium, indicating that the wild-type whiH gene promotes this hypercoiling, and is therefore likely to be active during aerial hyphal growth, at least in whiA and whiB mutants. However, this situation in the mutants may not reflect a normal activity: whiH single mutants show a degree of coiling similar to that of developing wild-type aerial hyphae.

WhiH resembles a family of DNA-binding regulatory proteins responsive to carboxylate-containing intermediates of carbon metabolism (Ryding et al., 1998). It has therefore been suggested that WhiH may sense some such metabolic intermediate, whose concentration changes during aerial growth (particularly at growth cessation), giving rise to two forms of WhiH: the form whose proportion increases later can be designated WhiH*. It is possible that later events are regulated by or indirectly require WhiH*. These would include increased expression of genes required for sporulation septation and for orchestration of the partitioning of DNA after the final one or two rounds of replication. Possibly, the level of $\mathrm{WhiH}^{*}$ may be important in transmitting information about whiA/whiB-dependent cessation of tip growth to genes whose up-regulation mediates subsequent events. On this view, whiH would provide a point of information exchange to coordinate the whiG-dependent regulatory 'cascade' (of which whiH is a part) with the apparently whiG-independent 'cascade' represented by whiB (Soliveri et al., 1992) and, perhaps, whiA (J. A. Aínsa \& K. F. Chater, unpublished). 


\section{ACKNOWLEDGEMENTS}

We thank N. J. Ryding for providing strains and information prior to publication, R. Losick for hosting K.F. for a brief period in his laboratory, E. A. Angert for demonstrating fluorescence microscopy techniques, and D. A. Hopwood, G. H. Kelemen and T. Kieser for helpful comments on the manuscript. K.F. was supported by grants from the BBSRC's Commitment and Determination programme (CAD 04380) and the EC (fellowship no. BIO4CT965134). K.F.C. was supported in part by the John Innes Foundation, and K.C.F. and K.F.C. were supported by a competitive strategic grant to the John Innes Centre from the BBSRC. In the context of this special issue of Microbiology to honour David Hopwood on his (formal) retirement, we wish to pay tribute to the key part that he played in this work by providing one of us (K.F.C) with his original collection of whi mutants some 30 years ago, and by his constant encouragement ever since.

\section{REFERENCES}

Bibb, M. J., Janssen, G. R. \& Ward, J. M. (1985). Cloning and analysis of the promoter region of the erythromycin resistance gene (ermE) of Streptomyces erythraeus. Gene 41, E357-E368.

Chater, K. F. (1972). A morphological and genetic mapping study of white colony mutants of Streptomyces coelicolor A3(2). J Gen Microbiol 72, 9-28.

Chater, K. F. (1975). Construction and phenotypes of double sporulation deficient mutants in Streptomyces coelicolor A3(2). J Gen Microbiol 87, 312-325.

Chater, K. F. (1989). Sporulation in Streptomyces. In Regulation of Prokaryotic Development, pp. 277-299. Edited by I. Smith, R. A. Slepecky \& P. Setlow. Washington, DC: American Society for Microbiology.

Chater, K. F. (1993). Genetics of differentiation in Streptomyces. Annu Rev Microbiol 47, 685-713.

Chater, K. F. (1998). Taking a genetic scalpel to the Streptomyces colony. Microbiology 144, 1465-1478.

Chater, K. F. \& Losick, R. (1997). Mycelial life style of Streptomyces coelicolor A3(2) and its relatives. In Bacteria as Multicellular Organisms, pp. 149-182. Edited by J. A. Shapiro \& M. Dworkin. New York: Oxford University Press.

Chater, K. F., Bruton, C. J. \& Suarez, J. E. (1982). The expression of Streptomyces and Escherichia coli drug-resistance determinants cloned into the Streptomyces phage $\phi \mathrm{C} 31$. Gene 19, 21-32.

Chater, K. F., Bruton, C. J., Plaskitt, K. A., Buttner, M. J., Méndez, C. \& Helmann, J. D. (1989). The developmental fate of S. coelicolor hyphae depends on a gene product homologous with the motility $\sigma$ factor of B. subtilis. Cell 59, 133-143.

Davis, N. K. \& Chater, K. F. (1990). Spore colour in Streptomyces coelicolor A3(2) involves the developmentally regulated synthesis of a compound biosynthetically related to polyketide antibiotics. Mol Microbiol 4, 1679-1691.

Davis, N. K. \& Chater, K. F. (1992). The Streptomyces coelicolor $w h i B$ gene encodes a small transcription factor-like protein dispensable for growth but essential for sporulation. Mol Gen Genet 232, 351-358.

Donachie, W. D. (1993). The cell cycle of Escherichia coli. Annu Rev Microbiol 47, 199-230.

Hanahan, D. (1983). Studies on transformation of Escherichia coli with plasmids. J Mol Biol 166, 557-580.

Hilleman, D., Pühler, A. \& Wohlleben, W. (1991). Gene disruption and gene replacement in Streptomyces via single stranded DNA transformation of integration vectors. Nucleic Acids Res 19, $727-731$.

Hopwood, D. A., Wildermuth, H. \& Palmer, H. M. (1970). Mutants of Streptomyces coelicolor defective in sporulation. J Gen Microbiol 61, 397-408.

Hopwood, D. A., Bibb, M. J., Chater, K. F. \& 7 other authors (1985). Genetic Manipulation in Streptomyces: a Laboratory Manual. Norwich: John Innes Foundation.

Hughes, K. T., Gillen, K. L., Semon, M. J. \& Karlinsey, J. E. (1993). Sensing structural intermediates in bacterial flagellar assembly by export of a negative regulator. Science 262, 1277-1280.

Kelemen, G. H., Brown, G. L., Kormanec, J., Potúčková, L., Chater, K. F. \& Buttner, M. J. (1996). The positions of the sigma factor genes $w h i G$ and $s i g F$ in the hierarchy controlling the development of spore chains in the aerial hyphae of Streptomyces coelicolor A3(2). Mol Microbiol 21, 593-603.

Kelemen, G. H., Brian, P., Flärdh, K., Chamberlin, L. C., Chater, K. F. \& Buttner, M. J. (1998). Developmental regulation of transcription of whiE, a locus specifying the polyketide spore pigment in Streptomyces coelicolor A3(2). J Bacteriol 180, 2515-2521.

Kieser, T. \& Hopwood, D. A. (1991). Genetic manipulation of Streptomyces: new integrating vectors and methods for gene replacement. Methods Enzymol 204, 430-458.

Kieser, T. \& Melton, R. E. (1988). Plasmid pIJ699, a multi-copy positive-selection vector for Streptomyces. Gene 65, 83-91.

Kretschmer, S. (1982). Dependence of the mycelial growth pattern on the individually regulated cell cycle in Streptomyces granaticolor. Z Allg Mikrobiol 22, 335-347.

Kwak, J. \& Kendrick, K. E. (1996). Bald mutants of Streptomyces griseus that prematurely undergo key events of sporulation. $J$ Bacteriol 178, 4643-4650.

Losick, R. \& Shapiro, L. (1993). Checkpoints that couple gene expression to morphogenesis. Science 262, 1227-1228.

MacNeil, D. J., Gewain, K. M., Ruby, C. L., Dezeny, G., Gibbons, P. H. \& MacNeil, T. (1992). Analysis of Streptomyces avermitilis genes required for avermectin biosynthesis utilising a novel integrating vector. Gene 111, 61-68.

McVittie, A. (1974). Ultrastructural studies on sporulation in wildtype and white colony mutants of Streptomyces coelicolor. J Gen Microbiol 81, 291-302.

Méndez, C. \& Chater, K. F. (1987). Cloning of whiG, a gene critical for sporulation of Streptomyces coelicolor A3(2). J Bacteriol 169, 5715-5720.

Miguélez, E. M., Rueda, B., Hardisson, C. \& Manzanal, M. B. (1998). Nucleoid partitioning and the later stages of sporulation septum synthesis are closely associated events in the sporulating hyphae of Streptomyces carpinensis. FEMS Microbiol Lett 159, 59-62.

Oh, S.-H. \& Chater, K. F. (1997). Denaturation of circular or linear DNA facilitates targeted integrative transformation of Streptomyces coelicolor A3(2): possible relevance to other organisms. J Bacteriol 179, 122-127.

Park, J. T. (1996). The murein sacculus. In Escherichia coli and Salmonella: Cellular and Molecular Biology, pp. 48-57. Edited by F. C. Neidhardt and others. Washington, DC: American Society for Microbiology.

Plaskitt, K. A. \& Chater, K. F. (1995). Influences of developmental genes on localised glycogen deposition in colonies of a mycelial prokaryote, Streptomyces coelicolor A3(2): a possible interface between metabolism and morphogenesis. Philos Trans $R$ Soc Lond B Biol Sci 347, 105-121. 
Potúčková, L., Kelemen, G. H., Findlay, K. C., Lonetto, M. A., Buttner, M. J. \& Kormanec, J. (1995). A new RNA polymerase sigma factor, $\sigma^{\mathrm{F}}$, is required for the late stages of morphological differentiation in Streptomyces spp. Mol Microbiol 17, 37-48.

Prosser, J. I. \& Tough, A. J. (1991). Growth mechanics and growth kinetics of filamentous microorganisms. Crit Rev Biotechnol 10, 253-274.

Ryding, N. J. (1995). Analysis of sporulation genes in Streptomyces coelicolor A3(2). PhD thesis, University of East Anglia.

Ryding, N. J., Kelemen, G. H., Whatling, C. A., Flärdh, K., Buttner, M. J. \& Chater, K. F. (1998). A developmentally regulated gene encoding a repressor-like protein is essential for sporulation in Streptomyces coelicolor A3(2). Mol Microbiol 29, 343-357.

Sambrook, J., Fritsch, E. F. \& Maniatis, T. (1989). Molecular Cloning: a Laboratory Manual. Cold Spring Harbor, NY: Cold Spring Harbor Laboratory.

Schwedock, J., McCormick, J. R., Angert, E. A., Nodwell, J. R. \& Losick, R. (1997). Assembly of the cell division protein FtsZ into ladder-like structures in the aerial hyphae of Streptomyces coelicolor. Mol Microbiol 25, 847-858.
Soliveri, J., Brown, K. L., Buttner, M. J. \& Chater, K. F. (1992). Two promoters for the whiB sporulation gene of Streptomyces coelicolor A3(2) and their activities in relation to development. J Bacteriol 174, 6215-6220.

Tan, H., Yang, H., Tian, Y., Wu, W., Whatling, C. A., Chamberlin, L. C., Buttner, M. J., Nodwell, J. \& Chater, K. F. (1998). The Streptomyces coelicolor sporulation-specific $\sigma^{\text {whic }}$ form of RNA polymerase transcribes a gene encoding a ProX-like protein that is dispensable for sporulation. Gene 212, 137-146.

Wildermuth, H. (1970). Development and organisation of the aerial mycelium in Streptomyces. J Gen Microbiol 60, 43-50.

Wildermuth, H. \& Hopwood, D. A. (1970). Septation during sporulation in Streptomyces coelicolor. J Gen Microbiol 60, 57-59.

Yu, T.-W. \& Hopwood, D. A. (1995). Ectopic expression of the Streptomyces coelicolor whiE genes for polyketide spore pigment synthesis and their interaction with the act genes for actinorhodin biosynthesis. Microbiology 141, 2779-2791.

Received 9 March 1999; accepted 11 May 1999. 